\title{
Neuropathic pain in the general population: a systematic review of epidemiological studies.
}

\author{
VAN HECKE, O., AUSTIN, S.K., KHAN, R.A., SMITH, B.H. and \\ TORRANCE, N.
}

2014 


\title{
Neuropathic pain in the general population: a systematic review of epidemiological studies
}

O. van Hecke ${ }^{a}{ }^{\Uparrow}$, Sophie K. Austin b, Rafi A. Khan c, B.H. Smith a, N. Torrance a

a Medical Research Institute, University of Dundee, UK

$\mathrm{b}$ Foundation Year, Livingston Hospital, NHS Lothian, Livingston, UK

c Department of Anaesthesia, Ninewells Hospital and Medical School, Dundee, UK

\begin{abstract}
Most patients with neuropathic pain symptoms present and are managed in primary care, with only a minority being referred for specialist clinical assessment and diagnoses. Previous reviews have focused mainly on specific neuropathic pain conditions based in specialist settings. This is the first systematic review of epidemiological studies of neuropathic pain in the general population. Electronic databases were searched from January 1966 to December 2012, and studies were included where the main focus was on neuropathic pain prevalence and/or incidence, either as part of a specific neuropathic pain-related condition or as a global entity in the general population. We excluded studies in which data were extracted from pain or other specialist clinics or focusing on specific population subgroups. Twenty-one articles were identified and underwent quality assessment and data extraction. Included studies differed in 3 main ways: method of data retrieval, case ascertainment tool used, and presentation of prevalence/incidence rates. This heterogeneity precluded any meta-analysis. We categorised comparable incidence and prevalence rates into 2 main subgroups: (1) chronic pain with neuropathic characteristics (range 3-17\%), and (2) neuropathic pain associated with a specific condition, including postherpetic neuralgia (3.9-42.0/100,000 person-years [PY]), trigeminal neuralgia (12.6-28.9/100,000 PY), painful diabetic peripheral neuropathy (15.3-72.3/100,000 PY), glossopharyngeal neuralgia (0.2-0.4/100,000 PY). These differences highlight the importance of a standardised approach for identifying neuropathic pain in future epidemiological studies. A best estimate of population prevalence of pain with neuropathic characteristics is likely to lie between $6.9 \%$ and $10 \%$.
\end{abstract}

Keywords: Epidemiology Incidence Neuropathic pain Prevalence Systematic review

\section{Introduction}

Neuropathic pain is widely recognised as one of the most difficult pain syndromes to manage, and outcomes often are unsatisfactory. This is partly because the contribution of neuropathy to pain presenting in primary care may be unrecognized $[2,10]$ and there is evidence of suboptimal drug use in the treatment of neuropathic pain [12,36,37]. Epidemiological research in this area can be problematic, and the reasons for this are multifactorial: the lack of agreed, valid case definitions that truly reflect the condition(s) under consideration and that are feasible to apply in population-based studies; heterogeneous studies of variable quality, using different means of case ascertainment; and inclusion or exclusion of cases in which pain is not a primary presenting complaint [10]. Existing estimates of the general population prevalence therefore vary widely, and it is likely that more people experience neuropathic symptoms than have been diagnosed with a neuropathic painrelated condition [11].

Sponsorships or competing interests that may be relevant to content are disclosed at the end of this article.

Article history:

Received 21 August 2013

Received in revised form 29 October 2013 Accepted 19 November 2013

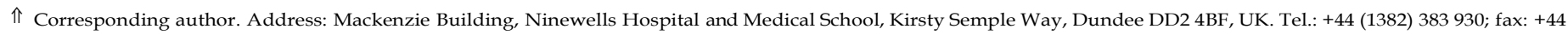
(1382) 383802.

E-mail address: o.vanhecke@dundee.ac.uk (O.van Hecke).
} 
Population-based estimates of neuropathic pain prevalence are important in order to determine resource requirements (clinical, financial, educational) for primary care, where most people with chronic pain are treated and managed, and to inform the targeting of treatment and prevention strategies [17]. As validated screening tools to identify neuropathic pain have been developed [3,34], these have enabled questionnaire-based epidemiological studies, and there is a growing body of literature exploring the epidemiology of neuropathic pain symptoms and conditions in the community. We conducted a systematic search and structured literature review of the epidemiology of neuropathic pain in the general population and aimed to provide, where possible, estimates of incidence and prevalence.

\section{Methods}

\subsection{Study selection}

A comprehensive search strategy was developed to identify all articles reporting population-based studies of the epidemiology of neuropathic pain (see Supplementary Materials). Electronic databases MEDLINE, EMBASE, ISI Web of Science, and CINAHL were searched from January 1966 to December 2012 for Englishlanguage articles that fulfilled the inclusion/ exclusion criteria. We included studies in which (1) the main focus was on neuropathic pain, either as part of a specific clinical condition or as a global clinical entity; (2) the core emphasis was on incidence and/or prevalence of neuropathic pain; and (3) the study sample was reasonably representative of the general population (thus including studies conducted in primary care/general practice populations where these approximated to general population samples). Article titles were reviewed to determine those that obviously did not meet the inclusion criteria and those that were required assessment in greater depth.

Excluded articles were those in which data were specifically obtained from pain or other specialist clinics, those that focused on specific population subgroups (eg, age, gender, occupation, ethnicity), and review articles (although reference lists of these were hand-searched for additional relevant studies). A grey literature search was performed after completion of the main search, seeking articles from conference proceedings and meetings on the ISI Web of Science database.

\subsection{Data extraction}

The database searches were conducted by S.A. and R.A.K. Titles were reviewed for possible inclusion, then abstracts examined, before the full-text version of the remaining articles were obtained. All authors were involved in various stages throughout this process and in the final selection and data extraction of included articles (Table 1). We extracted data on study characteristics (country, study design, and method of data retrieval); the sample size and number of patients with neuropathic pain in the sample population; case ascertainment instrument or tool used to identify the neuropathic pain condition; and the specific condition or disease associated with neuropathic pain.

\subsection{Quality assessment}

All articles that fully met the inclusion criteria were critically appraised using the STROBE checklist (STrengthening the Reporting of OBservational studies in Epidemiology) [40]. This is a structured, standardised checklist consisting of 22 items, each relating to the different sections in an article (ie, title, abstract, introduction, methods, results, discussion, and funding) with the main purpose being to improve the transparency of reporting in epidemiological observational research [40].

Quality factors based on key items of the STROBE quality checklist instrument were combined to form a modified checklist consisting of a total of 30 items with a simple point system. Each article was then given a total score out of 30 . The quality scores calculated for each article gave comparisons of the relative quality of included studies, a higher score indicating higher quality. A relatively low score did not necessarily imply poorquality research, as the score was a guide to the quality of reporting according to a specific (STROBE) checklist. 


\subsection{Analysis}

We aimed to compare the prevalence and incidence rates of specific neuropathic pain diagnoses, and/or of neuropathic pain as a global clinical entity.

3. Results

Fig. 1 shows the results of the article selection process.

\subsection{Characteristics of studies}

We identified 3826 articles from the electronic searches after removal of duplicates (Fig. 1). These articles were then reviewed by title, abstract, and full-text review and either included or excluded based on the selection criteria. Of these, 21 articles fulfilled the inclusion criteria and underwent quality assessment and data extraction and were included in the final review.

Fourteen studies were based in single European countries $[4,7,9,10,13-15,18,19,24,26,30,32,35]$, 4 studies were based in the United States $[6,22,23,42]$, and the remaining studies were each conducted in Brazil [8], Taiwan (China) [21], and Canada [38].

A wide variety of study designs was identified by the systematic review. There are 3 main ways in which the studies seemed to differ: first, in the method of data retrieval; secondly, in the case ascertainment tool used; and finally, in the presentation of the prevalence/incidence rates found. In order to make sense of the data, and to present results appropriately, it is important to take these factors into account.

\subsubsection{Data presentation of the prevalence/incidence rates}

Some studies focused on identifying rates of neuropathic pain that was a characteristic of chronic pain, whereas other studies reported rates of a clinical condition or disease associated with neuropathic pain. Due to the heterogeneity of included studies as noted earlier, the prevalence or incidence rates cannot be simply combined for meta-analysis. For this reason, we have categorised the incidence and prevalence rates into chronic pain with neuropathic characteristics or of neuropathic origin and neuropathic pain associated with a specific disease or condition.

\subsubsection{Method of data retrieval}

Data retrieval was either by electronic database searching or use of research questionnaires. Of the final 21 articles identified (Fig. 1), 14 studies were conducted using a database or primary care medical records, 6 studies were conducted by questionnaire or survey, and 1 study used a combination of both methods (Table $1)$.

\subsubsection{Case ascertainment of neuropathic pain}

A number of different ways of identifying cases were found. Generally, studies based on research questionnaires used validated case ascertainment tools such as the Douleur Neuropathique en 4 questions (DN4) [5] or the Self complete Leeds Assessment of Neuropathic Symptoms \& Signs (S-LANSS) [1], whereas studies that extracted data from databases relied on coding systems, such as the Oxford Medical Information System (OXMIS) classification [19] or the International Classification of Primary Care (ICPC) coding system [41] to identify cases.

As shown in Table 1, most studies included either incidence or prevalence rates, and 1 provided both [26]. Four studies did not provide specific incidence or prevalence rates $[6,7,9,30]$. However, these studies did provide sufficient information from which a prevalence or incidence rate could be calculated. 


\begin{tabular}{|c|c|c|c|c|c|c|c|c|c|}
\hline Study & Country & $\begin{array}{l}\text { Study design and method } \\
\text { of data retrieval }\end{array}$ & $\begin{array}{l}\text { Sample size } \\
\text { (response } \\
\text { rate) }\end{array}$ & $\begin{array}{l}\text { NeuP cases } \\
\text { identified }\end{array}$ & $\begin{array}{l}\text { NeuP case ascertainment method/ } \\
\text { instrument }\end{array}$ & NeuP condition & $\begin{array}{l}\text { Incidence } \\
\text { estimates } \\
{[95 \% \mathrm{Cl}]}\end{array}$ & $\begin{array}{l}\text { Prevalence } \\
\text { estimates } \\
{[95 \% \mathrm{CI}]}\end{array}$ & $\begin{array}{l}\text { Quality assessment } \\
\text { score (maximum } \\
30)^{*}\end{array}$ \\
\hline Bouhassira et al. (2008) & France & Postal survey & $\begin{array}{l}23,712 \\
(81.2 \%)\end{array}$ & 1631 & Self-administered DN4 & $\begin{array}{l}\text { Chronic pain with } \\
\text { neuropathic } \\
\text { characteristics (NeuP) }\end{array}$ & N/A & $\begin{array}{l}6.9 \% \\
{[6.6 \%-7.2 \%]}\end{array}$ & 26 \\
\hline Choo et al. (1997) & $\begin{array}{l}\text { United } \\
\text { States }\end{array}$ & $\begin{array}{l}\text { Automated medical } \\
\text { records review, claims } \\
\text { and pharmacy records }\end{array}$ & 250,000 & $\begin{array}{l}66 \text { (at } 30 \\
\text { days) } \\
37 \text { (at } 60 \\
\text { days) }\end{array}$ & $\begin{array}{l}\text { No standardised tool; clinical diagnosis } \\
\text { (recorded or likely) }\end{array}$ & $\begin{array}{l}\text { PHN (more than } 60 \text { days } \\
\text { after HZ) }\end{array}$ & $\begin{array}{l}0.007 \% \text { at } 60 \text { days } \\
(4.5 \% \text { of } \mathrm{HZV})\end{array}$ & N/A & 27 \\
\hline Davies et al. (2006) & UK & $\begin{array}{l}\text { Cross-sectional postal } \\
\text { survey and neurological } \\
\text { examination }\end{array}$ & $\begin{array}{r}8531 \\
(92.7 \%)\end{array}$ & $71^{i}$ & $\begin{array}{l}\text { Screening: } 1 x \text { item diabetic neuropathy } \\
\text { symptom score) } \\
\text { PDPN: clinical examination using } \\
\text { Toronto Clinical Scoring System }\end{array}$ & PDPN & N/A & $\begin{array}{l}\text { Overall } \\
\text { prevalence: } 0.8 \%\end{array}$ & 21 \\
\hline De Moraes Vieira (2012) & Brazil & $\begin{array}{l}\text { Cross-sectional interview } \\
\text { survey and physical } \\
\text { examination }\end{array}$ & 1597 & 157 & $\begin{array}{l}\text { Validated DN4 (Portuguese) and DN4- } \\
\text { based physical examination }\end{array}$ & NeuP & N/A & $\begin{array}{l}10 \% \text { No } 95 \% \text { CI } \\
\text { reported }\end{array}$ & 27 \\
\hline Dieleman et al. (2008) & Netherlands & $\begin{array}{l}\text { General practice research } \\
\text { database, cohort study }\end{array}$ & 362,693 & $\begin{array}{l}9135 \text { (all } \\
\text { NeuP) } \\
322(\mathrm{TN}) \\
4(\mathrm{GN})\end{array}$ & $\begin{array}{l}\text { Free text search and ICPC coding } \\
\text { determine diagnosis, then manual review } \\
\text { of electronic patient records }\end{array}$ & $\begin{array}{l}\text { Neuropathic pain } \\
\text { PHN } \\
\text { TN } \\
\text { GN }\end{array}$ & $\begin{array}{l}8.2 / 1000 \text { PY [8.0- } \\
8.4] \\
41.8 / 100,000 \mathrm{PY} \\
{[38.1-45.7]} \\
28.9 / 100,000 \mathrm{PY} \\
{[25.8-32.1]} \\
0.40 / 100,000 \mathrm{PY} \\
{[0.1-0.9]}\end{array}$ & N/A & 25 \\
\hline Gajria et al. (2011) & UK & $\begin{array}{l}\text { Computerised medical } \\
\text { records review }\end{array}$ & 105,877 & 1390 & $\begin{array}{l}33 \text { coded diagnosis of conditions in } \\
\text { medical records (READ codes) associated } \\
\text { with NeuP }\end{array}$ & Chronic pain with NeuP & $\mathrm{N} / \mathrm{A}$ & $\begin{array}{l}1.3 \% \\
\text { No } 95 \% \text { CI reported }\end{array}$ & 24 \\
\hline Gore et al. (2007) & UK & $\begin{array}{l}\text { General practice research } \\
\text { database }\end{array}$ & 1.9 million & 16,690 & $\begin{array}{l}\text { Coded diagnosis of conditions (OXMIS } \\
\text { and READ codes) }\end{array}$ & Predominate NeuP & N/A & $\begin{array}{l}0.9 \% \\
\text { No } 95 \% \text { CI reported }\end{array}$ & 17 \\
\hline Gustorff et al. (2007) & Austria & $\begin{array}{l}\text { Internet-based, } \\
\text { prospective } \\
\text { representative survey }\end{array}$ & 7707 & 260 & $\begin{array}{l}\text { Selected items from LANSS (part A) and } \\
\text { DN4 }\end{array}$ & Neuropathic pain & N/A & $\begin{array}{l}3.3 \% \\
\text { No } 95 \% \text { CI reported }\end{array}$ & 16 \\
\hline Hall et al. (2006) & UK & $\begin{array}{l}\text { General practice research } \\
\text { database }\end{array}$ & $>6.8$ million & $\begin{array}{l}\text { PHN: } 12,386 \\
\text { TN: } 8268 \\
\text { PLP: } 451 \\
\text { PDPN: } 4719\end{array}$ & $\begin{array}{l}\text { Coded diagnosis of conditions (OXMIS } \\
\text { and READ codes) }\end{array}$ & $\begin{array}{l}\text { PHN } \\
\text { TN } \\
\text { PLP } \\
\text { PDPN }\end{array}$ & $\begin{array}{l}40.2 / 100,000 \mathrm{PY} \\
{[39.5-40.9]} \\
26.8 / 100,000 \mathrm{PY} \\
{[26.2-27.4]} \\
1.5 / 100,000 \mathrm{PY} \\
{[1.3-1.6]} \\
15.3 / 100,000 \mathrm{PY} \\
{[14.9-15.7]}\end{array}$ & N/A & 21 \\
\hline Hall et al. (2008) & UK & $\begin{array}{l}\text { Computerised database of } \\
\text { primary care records } \\
\text { (THIN database) }\end{array}$ & $>2.9$ million & $\begin{array}{l}\text { PHN: } 1923 \\
\text { TN: } 1862 \\
\text { PLP: } 57 \\
\text { PDPN: } 1867\end{array}$ & $\begin{array}{l}\text { Coded diagnosis of conditions (OXMIS } \\
\text { and READ codes) }\end{array}$ & $\begin{array}{l}\text { PHN } \\
\text { TN } \\
\text { PLP } \\
\text { PDPN }\end{array}$ & $\begin{array}{l}\text { Age-standardised } \\
27.3 / 100,000 \mathrm{PY} \\
{[27.0-29.5]} \\
26.7 / 100,000 \mathrm{PY} \\
{[26.1-28.6]} \\
0.8 / 100,000 \mathrm{PY} \\
{[0.6-1.1]} \\
26.7 / 100,000 \mathrm{PY} \\
{[26.0-28.4]}\end{array}$ & $\mathrm{N} / \mathrm{A}$ & 19 \\
\hline Jih et al. (2009) & Taiwan & $\begin{array}{l}\text { National Health } \\
\text { Insurance electronic } \\
\text { database, retrospective } \\
\text { cohort study }\end{array}$ & 1 million & $\begin{array}{l}4543(30 \\
\text { days) } \\
2944(90 \\
\text { days })^{5}\end{array}$ & $\begin{array}{l}\text { No standardised tool; } \\
\text { ICPC code for HZ. } \\
\text { PHN defined as revisit }>90 \text { days after } \\
\text { onset or treatment }\end{array}$ & PHN & $\begin{array}{l}42.0 / 100,000 \mathrm{PY}^{\mathrm{k}} \\
\text { No } 95 \% \mathrm{CI} \\
\text { reported }\end{array}$ & N/A & 24 \\
\hline Katusic et al. (1990) & $\begin{array}{l}\text { United } \\
\text { States }\end{array}$ & Medical records & 222 & 75 & $\begin{array}{l}\text { Rushton and Olafson criteria for TN } \\
\text { Specialist review }\end{array}$ & $\mathrm{TN}$ & $\begin{array}{l}4.7 \text { per } 100,000 \\
\text { population }[3.6- \\
5.8]\end{array}$ & N/A & 20 \\
\hline Katusic et al. (1991) & $\begin{array}{l}\text { United } \\
\text { States }\end{array}$ & Medical records & 238 & 12 & $\begin{array}{l}\text { - No standardised definition } \\
\text { - Classification of } 4 \text { criteria in tex } \\
\text { - Specialist review }\end{array}$ & GN & $\begin{array}{l}0.8 \text { per } 100,000 \\
\text { population }[0.3- \\
1.2]\end{array}$ & N/A & 18 \\
\hline
\end{tabular}




\begin{tabular}{|c|c|c|c|c|c|}
\hline $\begin{array}{l}\text { Di Luzio Paparatti et al. } \\
\text { (1999) }\end{array}$ & Italy & $\begin{array}{l}\text { Medical records, } \\
\text { retrospective study }\end{array}$ & 98,508 & Not provided & $\begin{array}{l}\text { No standardised tool; PHN defined within } \\
\text { text (at least } 1 \text { month after HZV) }\end{array}$ \\
\hline MacDonald et al. (2000) & UK & $\begin{array}{l}\text { Database and } \\
\text { examination prospective } \\
\text { study }\end{array}$ & 100,230 & Not provided & Multiple methods \\
\hline Opstelten et al. (2002) & Netherlands & $\begin{array}{l}\text { Computerised medical } \\
\text { records review (GPRD) }\end{array}$ & $\sim 49,000$ & 837 & $\begin{array}{l}\text { No standardised tool; coded and free text } \\
\text { diagnosis of HZV and PHN }\end{array}$ \\
\hline Pierik et al. (2012) & \multicolumn{2}{|c|}{$\begin{array}{r}\text { Netherlands Retrospective cohort } \\
\text { study (2004-2008) }\end{array}$} & $\sim 165,000$ & $\begin{array}{l}195(\mathrm{PHN}) \\
75(\mathrm{TN}) \\
24(\text { facial } \\
\text { pain) }\end{array}$ & $\begin{array}{l}\text { ICPC code for varicella, and HZ and } \\
\text { noncoded information }\end{array}$ \\
\hline Torrance et al. (2006) & UK & $\begin{array}{l}\text { Cross-sectional postal } \\
\text { survey }\end{array}$ & $\begin{array}{l}3002 \\
(52.4 \%)\end{array}$ & 241 & S-LANSS \\
\hline Toth et al. (2009) & Canada & Telephone-based survey & $\begin{array}{l}1207 \\
(42.5 \%)\end{array}$ & Not provided & DN4 \\
\hline \multirow[t]{2}{*}{ Yawn et al. (2009) } & United & Postal survey, medical & 3575 & Clinical & Multiple: \\
\hline & States & $\begin{array}{l}\text { records, telephone } \\
\text { interview, and clinical } \\
\text { examination }\end{array}$ & $(61.0 \%)$ & $\begin{array}{l}\text { examination: } \\
\text { 75/205 } \\
\text { S-LANSS: } \\
315 / 3575 \\
\text { Berger } \\
\text { criteria: } \\
\text { 107/3575 } \\
\text { Self-reported: } \\
443 / 3575\end{array}$ & $\begin{array}{l}\text { 1. IASP criteria (clinical } \\
\text { examination) } \\
\text { 2. S-LANSS (postal) } \\
\text { 3. Berger criteria (medical records) }\end{array}$ \\
\hline
\end{tabular}

PHN

N/A

PHN

\section{PHN}

Overall (all ages) PHN TN Facial pain

\section{Pain of predominate} neuropathic origin Chronic pain with neuropathic symptoms $\mathrm{NeuP}$

\section{$0.09 \%$}

No $95 \%$ CI reported

17

$11 / 100,000$ / year [6.0-17.0] 0.7 per $1000[0.4-\quad 23$ [4.0-13,0] 0.7 per $1000[0.4$

$0.009 \%$

population/year N/A

47.5 per $10,000 \quad$ N/A [40.6-54.4]

$5.8 \%[5.0-6.6]$

$2.2 \%[1.71-2.71]$ $0.7 \%$ [0.41-0.97]

$\mathrm{N} / \mathrm{A}$

N/A

$17.9 \%[15.8 \%-$

$20.2 \%]$

Clinical

examination: $9.8 \%$ [6.2\%-13.4\%] S-LANSS: $8.8 \%$ [7.9\%-9.8\%] Berger criteria:
3.0\% [2.5\%-3.6\%] $3.0 \%[2.5 \%-3.6 \%]$ Self-reported: $13.6 \%]$

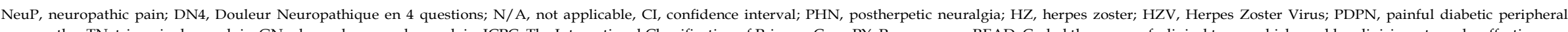

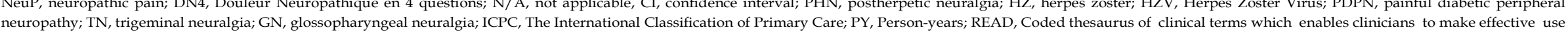

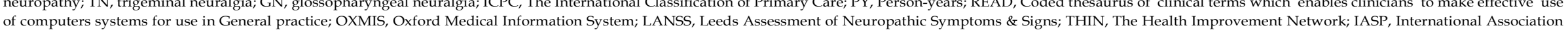
for the Study of Pain; GPRD, General Practice Research Database ; ICPC, International Classification of Primary Care; S-LANSS, Self-complete Leeds Assessment of Neuropathic Symptoms \& Signs.

* ${ }^{*}$ ased on STrengthening the Reporting of OBservational studies in Epidemiology (STROBE) checklist for reporting of observational studies (higher score correlating with higher quality).

Mixed PDPN included.

Prevalence of $26.4 \%$ of PDPN in those with type 2 diabetes who responded positively to screening question and attended for assessment.

${ }^{k}$ PHN incidence calculated at 90 days (3 months).

Original reported as 0.42 per $1000 \mathrm{PY}$.

Calculation extrapolated: (21.4\% of 408$) / 98,508$ 


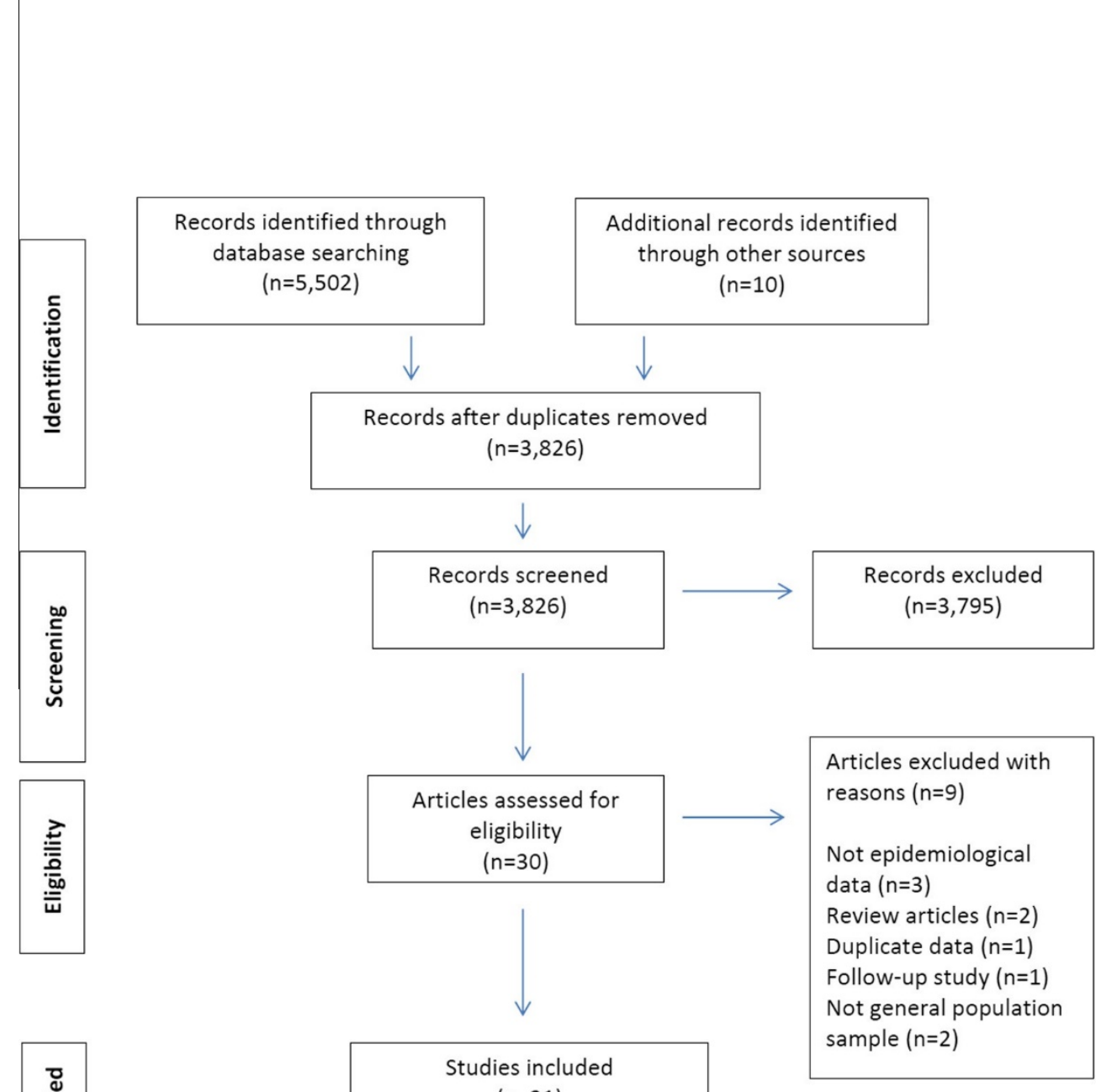

3.2. Results for studies on chronic pain with neuropathic characteristics or pain of predominantly neuropathic origin

\subsubsection{Prevalence}

Eight studies measured chronic pain with neuropathic (or 'predominantly neuropathic') characteristics $[4,8,13-15,35,38,42]$. The prevalence rates of neuropathic pain as a global clinical entity ranged from $0.9 \%$ to $17.9 \%$ (Table 2). All of these studies retrieved data by a postal questionnaire or telephone survey, with the exception of the studies by Gajria [13] (2011) and Gore et al. [14] (2007), which identified neuropathic cases from electronic databases. The DN4 case ascertainment tool was used by Bouhassira et al. [4] (2008), De Moraes Vieira et al. [8] (2012), and Toth et al. [38] (2009). The former 2 had comparable prevalence rates. Toth et al. [38] (2009) had a prevalence rate that was considerably higher. Gustorff et al. [15] (2007) used LANSS (part A), whereas Torrance et al. [35] (2006) and Yawn et al. [42] (2009) used the S-LANSS, and both studies found similar prevalence rates.

\subsubsection{Incidence}

Dieleman et al. [10] (2008) was the only study included. Reported incidence rates for a number of conditions associated with neuropathic pain were obtained, with an overall incidence rate in the general/study population of neuropathic pain of 8.2 per 1000 person-years [95\% confidence interval 8.0-8.4] (Table 2). 
A number of studies reported rates of a clinical condition or disease strongly associated with neuropathic pain $[6,7,9,10,18,19,21-24,26,30,32]$. The main conditions or diseases identified were postherpetic neuralgia (PHN), trigeminal neuralgia (TN), painful diabetic peripheral neuropathy (PDPN), and glossopharyngeal neuralgia (GN). Others conditions mentioned were phantom limb pain (PLP) and facial pain. Their prevalence and incidence rates are summarised in Tables 3 and 4 .

These studies were almost all database retrieval studies. A wide variety of case identification methods was used, often involving a number of different steps to verify the cases identified. For example, researchers in the study by Koopman et al. [24] used a 2-step process of case ascertainment: first, the researchers used the International Association for the Study of Pain (IASP) case definition criteria to search the database for different facial pain conditions associated with neuropathic pain and classed as either probable, possible, or no neuropathic pain; all probable cases were reviewed by a second medical doctor and discrepancies were arbitrated by a pain specialist. Secondly, additional information from the patients' medical records was sought to verify all possible cases.

\subsubsection{PHN}

Prevalence rates of PHN were reported in 2 included studies $[9,26]$. They ranged from $0.09 \%$ per annum and 0.7 per 1000 (Table 4). The study by Di Luzio Paparatti et al. [9] contained missing data. They reported that 408 of the 95,508 patients in their population had herpes zoster (HZ); however, only 322 of those were screened for complications. Of those screened, $21.4 \%$ had PHN. For the purposes of this analysis, we assumed that the rate for those screened was the same as for those not screened. The prevalence therefore was calculated by the equation $\left[\left(408^{*} 21.4\right) / 98,508\right]$.

The incidence rates of PHN were reported in 9 studies [6,10,18,19,21,24,26,30,32]. Note that some articles calculated incidence rates in percent of population per year [6,30,32], whereas others reported per 100,000 personyears $[10,18,19,21,24]$, and one reported number per population per year [26]. For this reason, direct comparisons could not be made among all studies. For those that were comparable, rates ranged from 3.9/100,000 person-years to $42.0 / 100,000$ person-years (Table 3).

The study by Opstelten et al. [30] (2002) was calculated from the data provided over a 5-year period, and the number in the total study population varied from year to year. Of the 837 individuals with $\mathrm{HZ}, 2.6 \%$ had persistent pain after 3 months. As the overall population was reported to be approximately 49,000, the rate was calculated by the equation: $\left.\left[\left(2.6^{*} 837\right) / 49,000\right)\right]$ over 5 years; dividing the answer by 5 found the incidence rate per population per year. A similar method was used in the study by Pierik et al. [32] to calculate the incidence.

The study by Choo et al. [6] (1997) had reported a prevalence of PHN among those who had experienced HZ in a general population said to number 250,000 . In fact, it was incidence that they calculated. There were 37 cases of PHN identified $>60$ days after $\mathrm{HZ}$ infection. The rate of PHN in the general population was recalculated using the equation $[37 / 250,000]$ and divided over 2 years. 
Table 2

Prevalence and incidence of chronic pain with neuropathic features and method of case ascertainment.

\begin{tabular}{|c|c|c|}
\hline Prevalence rate, $\%$ [95\% CI] & Author and year of publication & Case ascertainment tool \\
\hline $\begin{array}{l}6.9 \\
{[6.6-7.2]}\end{array}$ & Bouhassira etal. (2008) & DN4 \\
\hline $\begin{array}{l}10 \\
\text { No reported CI }\end{array}$ & De Moraes Vieira et al. (2012) & DN4 \\
\hline $\begin{array}{l}17.9 \\
\quad[15.8-20.2]\end{array}$ & Toth et al. (2009) & $\mathrm{DN} 4$ \\
\hline $\begin{array}{l}8.2 \\
{[7.2-9.2]}\end{array}$ & Torrance et al. (2006) & S-LANSS \\
\hline $\begin{array}{l}\text { Clinical examination: } 9.8 \% \\
\text { [6.2-13.4] } \\
\text { S-L ANSS: } 8.8 \% \\
\text { [7.9-9.8] } \\
\text { Berger criteria: } 3.0 \% \\
\text { [2.5-3.6] } \\
\text { Self-reported: } 12.4 \% \\
\text { [11.4-13.6] }\end{array}$ & Yawn etal. (2009) & $\begin{array}{l}\text { Multiple } \\
\text { - Clinical examination } \\
\text { - S-LANSS } \\
\text { - Berger criteria } \\
\text { - Self-reported }\end{array}$ \\
\hline 3.3No reported CI & Gustorff et al. (2007) & Selected items from LANSS (part A) and DN4, and additional questions \\
\hline Prevalence rate, $\%[95 \% \mathrm{CI}]$ & Author and year of publication & Cases coded from electronic databases \\
\hline 1.3No reported CI & Gajria et al. (2011) & Coded diagnosis (READ codes) \\
\hline $\begin{array}{l}0.9 \text { No reported } \mathrm{CI} \\
\text { Incidence rate }[95 \% \quad \mathrm{CI}]\end{array}$ & Gore et al. (2007) & Coded diagnosis (OXMIS and READ codes) \\
\hline $8.2 / 1000$ person-years[8.0-8.4] & Dieleman et al. (2008) & $\begin{array}{l}\text { Multiple } \\
\text { - Free text search and ICPC coding } \\
\text { - Manual review of electronic patient records }\end{array}$ \\
\hline
\end{tabular}

DN4, Doleur Neuropathique en 4 questions; OXMIS, Oxford Medical Information System; S-LANSS, Self-complete Leeds Assessment of Neuropathic Symptoms \& Signs; ICPC, International Classification of Primary Care.

Table 3

Incidence of neuropathic pain associated with a specific condition or disease.

\begin{tabular}{|c|c|c|c|}
\hline Condition or disease & Incidence rate* & Reference & Funding source \\
\hline \multirow[t]{9}{*}{ Postherpetic neuralgia } & 41.8 per $100,000 \mathrm{PY}$ & Dieleman et al. (2008) & 2 \\
\hline & 40.2 per $100,000 \mathrm{PY}$ & Hall et al. (2006) & 3 \\
\hline & 28.2 per $100,000 \mathrm{PY}$ & Hall et al. (2008) & 3 \\
\hline & 42.0 per $100,000 \mathrm{PY}^{t}$ & Jih et al. (2009) & 1 \\
\hline & 3.9 per $100,000 \mathrm{PY}$ & Koopman et al. (2009) & 2 \\
\hline & $11.0 / 100,000 /$ year & MacDonald et al. (2000) & 1 \\
\hline & $0.007 \% /$ population/year & Choo et al. (1997) & 2 \\
\hline & $0.009 \% /$ population/year & Opstelten et al. (2002) & 1 \\
\hline & $0.024 \% /$ population/year $\S$ & Pierik et al. $(2012)^{k}$ & 3 \\
\hline \multirow[t]{7}{*}{ Trigeminal neuralgia } & 28.9 per $100,000 \mathrm{PY}$ & Dieleman et al. (2008) & 2 \\
\hline & 26.8 per $100,000 \mathrm{PY}$ & Hall et al. (2006) & 3 \\
\hline & 27.3 per $100,000 \mathrm{PY}$ & Hall et al. (2008) & 3 \\
\hline & 12.6 per $100,000 \mathrm{PY}$ & Koopman et al. (2009) & 2 \\
\hline & 4.7 per 100,000 population & Katusic et al. (1990) & 1 \\
\hline & $8.0 / 100,000 /$ year & MacDonald et al. (2000) & 1 \\
\hline & $0.009 \% /$ population/yeare - & Pierik et al. $(2012)^{\mathrm{k}}$ & 3 \\
\hline \multirow[t]{3}{*}{ Glossopharyngeal neuralgia } & 0.4 per $100,000 P Y$ & Dieleman et al. (2008) & 2 \\
\hline & 0.8 per 100,000 population & Katusic et al. (1991) & 1 \\
\hline & 0.2 per $100,000 \mathrm{PY}$ & Koopman et al. (2009) & 2 \\
\hline \multirow[t]{2}{*}{ Phantom limb pain } & 1.5 per $100,000 \mathrm{PY}$ & Hall et al. (2006) & 3 \\
\hline & 0.8 per $100,000 \mathrm{PY}$ & Hall et al. (2008) & 3 \\
\hline \multirow[t]{3}{*}{ Painful diabetic peripheral neuropathy } & 72.3 per $100,000 \mathrm{PY}$ & Dieleman et al. (2008) & 2 \\
\hline & 15.3 per $100,000 \mathrm{PY}$ & Hall et al. (2006) & 3 \\
\hline & 27.2 per $100,000 \mathrm{PY}$ & Hall et al. (2008) & 3 \\
\hline
\end{tabular}

PY, person-years.

* Most incidence rates are calculated in person-years. This form of rate takes into consideration the dynamic nature of the population and allows direct comparisons in different populations.

${ }^{\mathrm{t}}$ Funding supported by: 1, government/charity/non-profit organisation; 2, educational grant from pharmaceutical industry, no industry authors; 3 , pharmaceutical industry funded and industry author included.

${ }^{\mathrm{t}}$ Extrapolated from 0.42/1000 PY.

${ }^{\S}$ Calculated: $195 / 165,000$ over 5 -year period $* 100(\%)$

${ }^{\mathrm{k}}$ Age-standardised incidence rate.

Calculated: 75/165,000 over 5-year period * $100(\%)$. 
Table 4

Prevalence of neuropathic pain associated with a specific condition or disease.

\begin{tabular}{lll}
\hline Condition/ disease......... & $\begin{array}{l}\text { Prevalence rate [95\% } \\
\text { confidence interval] }\end{array}$ & Reference \\
\hline Postherpetic neuralgia & $0.09 \%$ & Di Luzio \\
& No CI reported & Paparatti et al. \\
Trigeminal neuralgia & 0.7 per $1000[0.4-1.0]$ & MacDonald \\
Painful diabetic peripheral & 0.7 per $1000[0.4-1.0]$ & et al. \\
neuropathy & $0.8 \%{ }^{*}$ & Davies et al. \\
\end{tabular}

*Extrapolated: 71/8531*100; confidence intervals calculated.

3.3.2. $T N$

Incidence rates of TN were reported in 7 studies $[10,18,19,22,24,26,32]$. Comparable rates ranged from $12.6 / 100,000$ person-years to $28.9 / 100,000$ person-years (Table 3).

\subsubsection{PDPN}

The population prevalence of PDPN was calculated at $0.8 \%$, representing $26.4 \%$ of those with type 2 diabetes who responded positively to screening questions and attended clinical assessment (Table 4). Incidence rates of PDPN were reported in 3 studies [10,18,19]. Rates ranged from 15.3/100,000 person-years to 72.3/100,000 person-years (Table 3). PHN, TN, and PDPN had the highest incidence rates, compared to GN and PLP.

\subsection{Funding of included studies}

We identified 3 main sources of funding for the research. Some studies were funded by government, charity, or nonprofit organisations $[8,15,21-23,26,30,38]$; others had educational grants from industry but no authorship $[4,6,7,10,24,35,42]$; and the remainder were conducted and/or supported by pharmaceutical industry and had declared authorship $[9,13,14,18,19,32]$. There were no apparent systematic differences in study results associated with the source of funding.

\section{Discussion}

This is the first comprehensive systematic literature review of epidemiological studies of neuropathic pain in the general population. Rather than a traditional systematic review of randomised controlled trials, this review is based on observational studies within the general population using recently standardised methodology [31,40]. The heterogeneity between the identified studies precluded meta-analysis and calculation of combined estimates of prevalence and/or incidence rates of neuropathic pain. Nevertheless, we are able to draw comparisons between different studies and conditions and to consider these in the context of the relative quality, strengths and weaknesses, and study designs and reporting.

\subsection{Main findings}

Overall, the prevalence of neuropathic pain as a feature of chronic pain (or pain of predominantly neuropathic origin) was examined by 8 studies and ranged from 1\% to 17.9\% (Table 21). In studies that used questionnaire-based case ascertainment tools such as DN4 and S-LANSS, the range of prevalence estimates was wide (3.3-17.9\%). However, including only studies that administered case ascertainment tools precisely as they were designed and validated, the range of prevalence rates was much narrower $(6.9-10 \%)$ [4,8,35,42].

Gustorff et al. [15] utilised the LANSS (Part A) along with additional pain questions and some specific exclusion criteria, limiting their analysis to people with a known underlying neuropathic pain diagnosis (people with exclusive joint pain or reported rheumatism, migraine, fibromyalgia, visceral pain, and/or satisfying 
response to over-the-counter analgesic medication were excluded from further interview, as well as people suffering from an undifferentiated cause as the main cause of their pain); this explains their estimate of the lowest prevalence of neuropathic pain at $3.3 \%$.

In contrast, Toth et al. [8] included all respondents who reported "daily or near daily pain" from telephone surveys of the general population in a Canadian province (finding a prevalence of 38\%). Respondents then completed the DN4, with a resultant highest prevalence estimate of neuropathic pain at $17.9 \%$. This is likely an overestimate due to the screening question used to identify the presence of pain or selection bias in the underlying population. Other studies, utilising data obtained from computerised medical records, found a much lower overall prevalence of around 1\% [13,14]. This lower rate likely reflects differences in case ascertainment, and in particular that electronic records can only identify patients who have presented to primary care and had a diagnosis recorded by the attending physician.

When we examined studies of specific neuropathic pain conditions, most of them reported incidence rates per person-years, which is a useful parameter for comparing rates, although less clinically useful as a direct indicator of the number of cases likely to arise in a specific population during a specific period. Koopman et al. [24] consistently reported lower incidence rates of PHN, TN, and GN (Table 3) than the other studies, and this is mostly likely due to differences in case ascertainment. This can be illustrated by comparing this study with that reported by Dieleman et al. [10]. Both of these studies were conducted in consecutive years and both used the IPCI database in the Netherlands, yet they found very different incidence rates. For example, looking at TN, Koopman et al. reported an incidence rate of 12.6/100,000 person-years, whereas Dieleman et al. reported a rate of 28.9/100,000 person years. Koopman et al. accessed additional information when identifying the cases, and the authors were able to identify and then to exclude misclassified cases (false-positive cases) from the analysis. As a result, the authors suggest their estimate of the incidence of TN was less likely to be exaggerated due to misclassification.

Because of the heterogeneity of included studies, it is not possible to calculate a valid weighted prevalence or incidence for any neuropathic pain condition. Therefore, based on the quality, methods, and other characteristics of the studies we reviewed, and acknowledging this heterogeneity, a best-estimate range of comparable incidence rates for PHN is potentially narrower (28.2-42.0/100,000 person-years) [10,18,19,21], and similar for TN (26.828.9/100,000 person-years) [10,18,19], GN (0.4-0.8/100,000 person-years) [10,23], and PLP $(0.8-1.5 / 100,000$ person-years) [18,19]. The incidence range of PDPN remains wide $(15.3-72.3 / 100,000$ person-years) $[10,18,19]$ despite comparable incidence rates for PHN and TN. Irrespective of these differences, it seems clear that GN and PLP occur much less frequently than PDPN or PHN.

\section{2. $\quad$ Other findings}

It was surprising that some articles did not provide a working definition for neuropathic pain as a starting point. The articles that did include a definition cited the IASP (Merskey and Bogduk) definition [27]: chronic pain that is "initiated or caused by a primary lesion or dysfunction in the nervous system." This definition has since been superseded as pain "arising as a direct consequence of a lesion or disease affecting the somatosensory system" $[20,25]$.

Although case ascertainment tools were used by a number of studies, these were neither designed to nor did they claim to make a diagnosis of definite neuropathic pain; rather they were validated in identifying pain with neuropathic characteristics [5]. Some studies were clear in their acknowledgment of this in describing the cases $[4,35,38,42]$, but other studies did not distinguish between neuropathic features and neuropathic pain $[8,15]$.

In studies based on reviews of medical records, the identification of cases relies on accurate diagnoses of neuropathic pain (conditions) by the physician, who then must enter details accurately, using an accurate coding system and an accurate method of retrieving these codes. Rates calculated from medical records also may be affected by severity of pain or disease, as patients with more severe cases are more likely to seek medical attention [37]. Factors that may affect incidence and prevalence rate estimates (in general) are summarised in Table 5.

Although there are published guidelines on the assessment of neuropathic pain [17], the lack of an agreed-upon specific diagnostic tool has resulted in a proposed grading system of definite, probable, and possible neuropathic pain [20]. This is based on a combination of plausible distribution and history of pain, other positive confirmatory tests, and clinical investigation(s) by a pain specialist or neurologist [39]. For large epidemiological studies, the 
grading of probable or definite neuropathic pain is not practicable to achieve, and at best the case definitions included in this review may approximate possible neuropathic pain. Existing Special Interest Group on Neuropathic Pain (NeuPSIG) guidelines on neuropathic pain assessment advise that existing screening tools require further validation before they can be completely useful for epidemiological studies in general population samples [16]. Further research should look to explore how the findings of these questionnaire-based screening instruments/case ascertainment tools relate to specialist clinical examinations in a general population sample.

Neuropathic pain is associated with a number of clinical conditions and diseases, but only a small number of them were examined in the articles identified for inclusion in this review. Particular aetiologies such as PHN were more commonly studied, whereas others such as spinal cord injury [33] were not studied in a general population context. Published studies of the epidemiology of these less common subgroups tended to be conducted in small samples, in specialist settings, or in secondary care, providing an indication of their likelihood in these subgroups, but not of the overall population burden or risk.

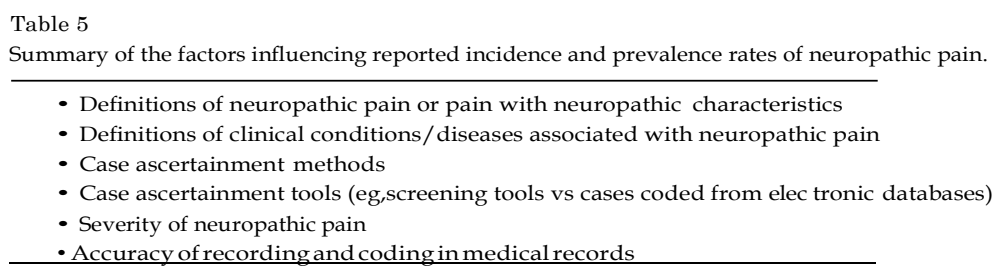

\subsection{Strengths and weaknesses}

The strengths of this review are in its strict systematic approach and consistency in application of the criteria throughout. A thorough search strategy was developed. The studies included were all of medium to high quality.

Although a number of the screening tools that identify pain with neuropathic characteristics have been translated and validated for use in other languages [29], a limitation in this review was the exclusion of nonEnglish-language articles, as translation was beyond the available resources.

Unlike other systematic reviews [28,31], there is no recognised quality checklist pertaining to observational studies within the general population. Hence a quality checklist was developed from a well-used standard tool specifically for epidemiological studies [40]. Although it is not a standard instrument for systematic reviews, it does provide a reasonable indicator of quality and validity, allowing rejection of articles or studies of low quality and comparison between studies.

\subsection{Implications for research and clinical practice}

As the use of screening tools within the published literature increases, it may be possible to restrict a future review to a specified case ascertainment tool, eg, DN4 or S-LANSS. The studies included would therefore have greater consistency, would have similar features in common, and would be more comparable when analysing the results.

Now that there is an agreed-upon definition of neuropathic pain, it is important to acknowledge its limitations and the application of this within large epidemiological studies. There is also a good argument that neuropathic pain should be considered as a Medical Subject Headings (MeSH) term in databases.

\subsection{Conclusions}

A comprehensive literature search and systematic review was performed on the epidemiology of neuropathic pain in the general population. A range of incidence and prevalence rates has been identified. The lack of consensus on a definition, diagnostic criteria, and appropriate and consistent use of the screening tools are reflected by the varying methods and results of the studies included in this review. Future epidemiological studies into neuropathic pain within the community should take note of these factors, using a standardised approach for identifying pain with possible neuropathic features. 


\section{Conflict of interest}

B.S. and N.T. have previously received an unrestricted educational grant from Pfizer UK Ltd. for research on neuropathic pain. B.S. has received occasional lecture and consultancy fees, on behalf of his institution, from Pfizer and Napp. The authors otherwise assert no personal pecuniary or other conflict of interest in the writing of this article. No writing assistance or external funding was obtained for this study.

\section{Appendix A. Supplementary data}

Supplementary data associated with this article can be found, in the online version, at http:/ / dx.doi.org/10.1016/j.pain.2013.11.013.

\section{References}

[1] Bennett MI, Smith BH, Torrance N, Potter J. The S-LANSS score for identifying pain of predominantly neuropathic origin: Validation for use in clinical and postal research. J Pain 2005;6:149-58.

[2] Bennett MI, Smith BH, Torrance N, Lee AJ. Can pain can be more or less neuropathic? Comparison of symptom assessment tools with ratings of certainty by clinicians. PAIN® 2006;122:289-94.

[3] Bennett MI, Attal N, Backonja MM, Baron R, Bouhassira D, Freynhagen R, Scholz J, Tölle TR, Wittchen HU, Jensen TS. Using screening tools to identify neuropathic pain. PAIN® 2007;127:199-203.

[4] Bouhassira D, Lanteri-Minet M, Attal N, Laurent B, Touboul C. Prevalence of chronic pain with neuropathic characteristics in the general population. PAIN® 2008;136:380-7.

[5] Bouhassira D, Attal N, Alchaar H, Boureau F, Brochet B, Bruxelle J, Cunin G, Fermanian J, Ginies P, GrunOverdyking A, Jafari Schluep H, Lantéri-Minet M, Laurent B, Mick G, Serrie A, Valade D, Vicaut E. Comparison of pain syndromes associated with nervous or somatic lesions and development of a new neuropathic pain diagnostic questionnaire (DN4). PAIN ${ }^{\circledR}$ 2005;114:29-36.

[6] Choo PW, Galil K, Donahue JG, Walker AM, Spiegelman D, Platt R. Risk factors for postherpetic neuralgia. Arch Intern Med 1997;157:1217-24.

[7] Davies M, Brophy S, Williams R, Taylor A. The prevalence, severity, and impact of painful diabetic peripheral neuropathy in type 2 diabetes. Diabetes Care 2006;29:1518-22.

[8] De Moraes Vieira EB, Garcia JB, Da Silva AA, Mualem Araujo RL, Jansen RC. Prevalence, characteristics, and factors associated with chronic pain with and without neuropathic characteristics in Sao Luis, Brazil. J Pain Symptom Manage 2012;44:239-51.

[9] Di Luzio Paparatti U, Arpinelli F, Visona G. Herpes zoster and its complications in Italy: An observational survey. J Infect 1999;38:116-9.

[10] Dieleman JP, Kerklaan J, Huygen FJ, Bouma PA, Sturkenboom MC. Incidence rates and treatment of neuropathic pain conditions in the general population. PAIN ${ }^{\circledR} 2008 ; 137: 681-8$.

[11] Dworkin RH. An overview of neuropathic pain: Syndromes, symptoms, signs, and several mechanisms. Clin J Pain 2002;18:343-9.

[12] Dworkin RH, Panarites CJ, Armstrong EP, Malone DC, Pham SV. Is treatment of postherpetic neuralgia in the community consistent with evidence-based recommendations? PAIN ${ }^{\circledR}$ 2012;153:869-75.

[13] Gajria C, Murray J, Birger R, Banarsee R, Bennett DL, Tan K, Field M, Rice AS, Majeed A. Identification of patients with neuropathic pain using electronic primary care records. Inform Prim Care 2011;19:83-90.

[14] Gore M, Dukes E, Rowbotham DJ, Tai K, Leslie D. Clinical characteristics and pain management among patients with painful peripheral neuropathic disorders in general practice. Eur J Pain 2007;11:652-64.

[15] Gustorff B, Dorner T, Likar R, Grisold W, Lawrence K, Schwarz F, Rieder A. Prevalence of self-reported neuropathic pain and impact on quality of life: A prospective representative survey. Acta Anaesthesiol Scand 2008;52:132-6.

[16] Haanpää M, Attal N, Backonja M, Baron R, Bennett M, Bouhassira D, Cruccu G, Hansson P, Haythornthwaite JA, Iannetti GD, Jensen TS, Kauppila T, Nurmikko TJ, Rice AS, Rowbotham M, Serra J, Sommer C, Smith BH, Treede RD. NeuPSIG guidelines on neuropathic pain assessment. PAIN ${ }^{\circledR} 2011 ; 152: 14-$ 27. 
[17] Haanpää ML, Backonja MM, Bennett MI, Bouhassira D, Cruccu G, Hansson PT, Jensen TS, Kauppila T, Rice AS, Smith BH, Treede RD, Baron R. Assessment of neuropathic pain in primary care. Am J Med 2009;122:S13-21.

[18] Hall GC, Carroll D, McQuay HJ. Primary care incidence and treatment of four neuropathic pain conditions: A descriptive study, 2002-2005. BMC Fam Pract 2008;9:26.

[19] Hall GC, Carroll D, Parry D, McQuay HJ. Epidemiology and treatment of neuropathic pain: The UK primary care perspective. PAIN ${ }^{\circledR}$ 2006;122:156-62.

[20] Jensen TS, Gebhart GF. New pain terminology: A work in progress. PAIN® 2008;140:399-400.

[21] features and costs of herpes zoster in Taiwan: A national study 2000 to 2006. Acta Derm Venereol 2009;89:612-6.

[22] Katusic S, Beard CM, Bergstralh E, Kurland LT. Incidence and clinical features of trigeminal neuralgia, Rochester, Minnesota, 1945-1984. Ann Neurol 1990;27:89-95.

[23] Katusic S, Williams DB, Beard CM, Bergstralh E, Kurland LT. Incidence and clinical features of glossopharyngeal neuralgia, Rochester, Minnesota, 1945- 1984. Neuroepidemiology 1991;10:266-75.

[24] Koopman JS, Dieleman JP, Huygen FJ, de Mos M, Martin CG, Sturkenboom MC. Incidence of facial pain in the general population. PAIN ${ }^{\circledR} 2009 ; 147: 122-7$.

[25] Loeser JD, Treede R. The Kyoto protocol of IASP Basic Pain Terminology. PAIN ${ }^{\circledR ~ 2008 ; 137: 473-7 . ~}$

[26] MacDonald BK, Cockerell OC, Sander JW, Shorvon SD. The incidence and lifetime prevalence of neurological disorders in a prospective community- based study in the UK. Brain 2000;123:665-76.

[27] Merskey H, Bogduk N. Descriptions of chronic pain syndromes and definitions of pain terms. Classification of chronic pain. Seattle: IASP Press; 1994.

[28] Moher D, Liberati A, Tetzlaff J, Altman DG, The PRISMA, Group. Preferred reporting items for systematic reviews and meta-analyses: The PRISMA statement. J Clin Epidemiol 2009;62:1006-12.

[29] NeuPSIG (Special Interest Group on Neuropathic Pain). Neuropathic pain screening tools. Available at: http:/ / www.neupsig.de/screeningtools.html. Accessed 13.08.13.

[30] Opstelten W, Mauritz JW, de Wit NJ, van Wijck AJ, Stalman WA, van Essen GA. Herpes zoster and postherpetic neuralgia: Incidence and risk indicators using a general practice research database. Fam Pract 2002;19:471-5.

[31] Petticrew M, Roberts H. How to appraise the studies: An introduction to assessing study quality. Systematic reviews in the social sciences: A practical guide. Oxford: Blackwell Publishing; 2006. p. 125-163.

[32] Pierik JG, Gumbs PD, Fortanier SA, Van Steenwijk PC, Postma MJ. Epidemiological characteristics and societal burden of varicella zoster virus in the Netherlands. BMC Infect Dis 2012;12:110.

[33] Siddall PJ, McClelland JM, Rutkowski SB, Cousins MJ. A longitudinal study of the prevalence and characteristics of pain in the first 5 years following spinal cord injury. PAIN ${ }^{\circledR ~ 2003 ; 103: 249-57 . ~}$

[34] Smith BH, Torrance N, Ferguson JA, Bennett MI, Serpell MG, Dunn KM. Towards a definition of refractory neuropathic pain for epidemiological research. An international Delphi survey of experts. BMC Neurol 2012;12:29.

[35] Torrance N, Smith BH, Bennett MI, Lee AJ. The epidemiology of chronic pain of predominantly neuropathic origin. Results from a general population survey. J Pain 2006;7:282-9.

[36] Torrance N, Smith BH, Watson MC, Bennett MI. Medication and treatment use in primary care patients with chronic pain of predominantly neuropathic origin. Fam Pract 2007;24:481-5.

[37] Torrance N, Ferguson JA, Afolabi E, Bennett MI, Serpell MG, Dunn KM, Smith BH. Neuropathic pain in the community: More under-treated than refractory? PAIN ${ }^{\circledR} 2013 ; 154: 690-9$.

[38] Toth C, Lander J, Wiebe S. The prevalence and impact of chronic pain with neuropathic pain symptoms in the general population. Pain Med 2009;10:918-29.

[39] Treede RD, Jensen TS, Campbell JN, Cruccu G, Dostrovsky JO, Griffin JW, Hansson P, Hughes R, Nurmikko T, Serra J. Neuropathic pain: Redefinition and a grading system for clinical and research purposes. Neurology 2008;70:1630-5.

[40] Von Elm E, Altman DG, Egger M, Stuart J, Pocock SJ, Gøtzsche PC, Vandenbroucke JP. Strengthening the reporting of observational studies in epidemiology (STROBE) statement: Guidelines for reporting observational studies. BMJ 2007;335:7624. 
[41] WONCA International Classification Committee. International classification of primary care. Oxford University

Press;

1987.

Available

at:

http:// www.globalfamilydoctor.com/groups/WorkingParties/wicc.aspx. Accessed 01.07.13.

[42] Yawn BP, Wollan PC, Weingarten TN, Watson JC, Hooten WM, Melton 3rd LJ. The prevalence of neuropathic pain: Clinical evaluation compared with screening tools in a community population. Pain Med 2009;10:586-93. 